\title{
PERFIL DOS PACIENTES DE UM CENTRO ODONTOLÓGICO DE REFERÊNCIA PARA PACIENTES ESPECIAIS EM MATO GROSSO ATENDIDOS EM AMBIENTE HOSPITALAR
}

\author{
PROFILE OF PATIENTS OF AN ODONTOLOGICAL REFERENCE CENTER FOR SPECIAL \\ PATIENTS IN MATO GROSSO SERVED IN HOSPITAL ENVIRONMENT
}

\section{Lorraynne dos Santos LARA'; Romualdo Prata VIDAL2; Aurélio Rosa da SILVA JUNIOR; Cristiane Loreda GARCIA4; Luiz Evaristo Ricci VOLPATO 5}

1 - Acadêmica do Curso de Odontologia pela Universidade de Cuiabá (UNIC).

2 - Cirurgião-Dentista, Especialista em Endodontia e Odontologia para Pacientes Especiais.

3 - Doutorando em Ciências Odontológicas Integradas - UNIC.

4 - Mestranda em Ciências Odontológicas Integradas - UNIC.

5 - Professor do Programa de Mestrado e Doutorado em Ciências Odontológicas Integradas - UNIC.

\section{RESUMO}

Objetivo: Este trabalho teve como objetivo traçar o perfil dos pacientes especiais de um centro odontológico de referência atendidos em ambiente hospitalar. Material e método: A pesquisa foi realizada através da análise de prontuários de pacientes atendidos nos anos de 2016, 2017 e 2018 no Centro Estadual de Odontologia para Pacientes Especiais (CEOPE), Cuiabá- MT. Os parâmetros analisados foram: sexo, idade, condição clínica prévia (doenças metabólicas; condição de déficit cognitivo; doenças psíquicas; sequelas físicas permanentes e má formação congênita), tipo da anestesia (geral, local, não realizada), procedimentos realizados (invasivos; não invasivos; ambos) e se recebe ou não cuidados em regime de home care. Resultados: Os pacientes atendidos em ambiente hospitalar eram em sua maioria do sexo masculino 28 (60,86\%). A faixa etária mais prevalente foi adulta (entre 19 e 59 anos) com 31 pacientes (67,39\%). A condição clínica prévia mais frequente entre os pacientes foi doenças crônicas e síndromes metabólicas com 14 pacientes (30,43\%). A maior parte dos pacientes não dispunha de cuidados em regime de home care $43(93,47 \%)$. O tipo da anestesia mais utilizado foi a anestesia geral, em 29 pacientes (63,04\%). Dos procedimentos realizados, houve prevalência de procedimentos invasivos, em 30 casos $(65,21 \%)$. Conclusões: O estudo revelou que o perfil do paciente com necessidade especial, atendido em ambiente hospitalar é adulto, do sexo masculino, com doenças crônicas e síndromes metabólicas para a realização de procedimentos invasivos sob anestesia geral.

Palavras-chave: Equipe Hospitalar de Odontologia; Odontologia; Pessoas com Deficiência; Assistência Odontológica; Unidade Hospitalar de Odontologia.

\section{INTRODUÇÃO}

Pessoas com deficiências ou outras necessidades especiais representam uma parcela significativa da sociedade e essa parcela vem aumentando a cada dia. Esse grupo de indivíduos apresenta maiores necessidades odontológicas que a população em geral. Esse fato pode estar relacionado à maior dificuldade de manter cuidados preventivos adequados e acesso limitado aos centros de saúde ${ }^{1}$. A maioria desses pacientes não tem capacidade e consciência para realizar o autocuidado e precisam contar com seus pais ou cuidadores para cuidados gerais. Frequentemente os cuidados de saúde bucal são ignorados nesses pacientes ${ }^{2}$.

Como a condição sistêmica desses pacientes geralmente é complexa, muitas vezes esgotam seus prestadores de cuidados e certos cuidadores não possuem conhecimento essencial para detectar potenciais problemas dentários. Assim, necessidades de tratamento odontológico tornam-se a principal preocupação de saúde médica não atendida entre esses pacientes².
Além disso, o risco de complicação infecciosa é elevado nesta população, que tem uma maior prevalência de doenças sistêmicas, como doença cardíaca congênita, imunodeficiência, diabetes ou próteses internas ${ }^{3}$.

Assim, muitas vezes o tratamento sob anestesia geral em ambiente hospitalar se torna indispensável para pacientes com deficiência mental moderada e severa, com o cognitivo baixo, comportamentos inadequados, que apresentam movimentos involuntários, alergia a anestésicos locais ou com doenças sistêmicas e com necessidade de tratamento odontológico acumulada que impossibilitem o atendimento odontológico ambulatorial. $\mathrm{O}$ tratamento em ambiente hospitalar tem como vantagem a possibilidade de executar todos os procedimentos em uma única sessão, com conforto para o paciente e profissional ${ }^{4}$.

Para gerenciar o elevado risco de complicação infecciosa nessa população, os cirurgiões-dentistas que trabalham no ambiente hospitalar frequentemente priorizam as extrações em relação 
ao tratamento conservador. Entretanto, a maioria dos pacientes com deficiências cognitivas ou mentais não consegue dominar o uso de próteses dentárias e não possui os recursos financeiros necessários para próteses fixas ou implantes. De forma que nesta população, sobretudo naqueles indivíduos com incapacidades neuromotoras, extrações múltiplas e edentulismo exacerbam problemas nutricionais ${ }^{3}$.

A Constituição Brasileira de 1988 tem a equidade como um dos princípios norteadores do Sistema Único de Saúde (SUS), juntamente com o acesso universal e a priorização da assistência. O Governo de Mato Grosso (MT) implantou em julho de 2005 o Centro Estadual de Odontologia para Pacientes Especiais (CEOPE) por meio da Lei 8.344/055. O CEOPE tem como objetivo garantir o acesso humanizado e assistência odontológica pública às pessoas com necessidades especiais. Desde então o referido centro tem oferecido assistência odontológica aos pacientes em ambiente ambulatorial bem como ambiente hospitalar em unidades parceiras ${ }^{5}$.

O objetivo deste trabalho foi traçar o perfil dos pacientes especiais de um centro público de referência de Mato Grosso atendidos em ambiente hospitalar.

\section{MATERIAIS E MÉTODOS}

Antes do início das coletas de dados, o projeto referente a esta pesquisa foi submetido à apreciação pelo Comitê de Ética em Pesquisa da Universidade de Cuiabá (CEP/UNIC), tendo recebido parecer favorável à sua realização sob o número 2.892.223.

Esta pesquisa envolveu riscos mínimos aos voluntários, uma vez que não foram realizados procedimentos clínicos, mas a busca em seus prontuários de dados relacionados ao sexo, idade, condição clínica prévia e tratamentos realizados em ambiente hospitalar. Foram incluídos no estudo todos os pacientes que iniciaram o tratamento nos anos de 2016, 2017 e 2018.

\section{Caracterização da população}

Foi realizado o levantamento do perfil dos pacientes especiais atendidos no Centro Estadual de Odontologia para Pacientes Especiais (CEOPE).

Essa pesquisa envolveu riscos mínimos aos voluntários, uma vez que não foram realizados procedimentos clínicos, mas a busca em seus prontuários de dados relacionados ao sexo, idade, condição clínica prévia e tratamentos realizados em ambiente hospitalar. Foram incluídos no estudo todos os pacientes que iniciaram o tratamento nos anos de 2016, 2017 e 2018.

\section{Critérios de inclusão}

Pacientes com necessidades especiais de cuidados de saúde sob tratamento no CEOPE que iniciaram o tratamento nos anos de 2016, 2017 e 2018 e se submeteram a procedimentos odontológicos em ambiente hospitalar.

\section{Critérios de exclusão}

Pacientes que se negaram a participar da pesquisa ou cujo prontuário não permitiu obtenção adequada dos dados procurados nesta pesquisa.

Após a obtenção dos dados, estes foram tabulados e, após análise descritiva, apresentados em forma de tabela com valores absolutos e relativos e figura.

Os pacientes foram agrupados em 4 grupos, de acordo com sua faixa etária: Crianças: de 0 a 12 anos; Jovens: de 13 a 18 anos; Adultos: de 19 a 59 anos; e Idosos: com 60 anos ou mais.

Segundo a condição clínica prévia, os pacientes foram agrupados em 5 grupos: Doenças Metabólicas; Condição de déficit cognitivo; Doenças psíquicas; Sequelas físicas permanentes; e Má formação congênita. Sendo consideradas as seguintes doenças em cada grupo:

a. Doenças crônicas e síndromes metabólicas: Insuficiência renal crônica, diabetes, cardiopatia, anemia, distúrbios de coagulação, hipertensão arterial, lúpus, Tumor de Wilms, Síndrome de Sanfilippo, Púrpura, encefalopatia bilirrubínica, alterações pulmonares, hipertireoidismo.

b. Condição de déficit cognitivo: Autismo, paralisia cerebral, hidrocefalia, retardo mental, microcefalia, neuropatia, atrofia muscular, craniostenose e sequelas de doenças inflamatórias do sistema nervoso central.

c. Doenças psíquicas: Esquizofrenia, epilepsia, hiperatividade, depressão, tratamento neurológico, Síndrome do Pânico, labirintite, enxaqueca, transtornos mentais, de desenvolvimento e comportamentais.

d. Sequelas físicas permanentes e alterações sensoriais: Sequela de acidente vascular encefálico, sequela de lesão por arma de fogo, sequela pós-cirurgia, sequela de acidente de trânsito, deficiente visual, deficiente auditivo e de fala, traumatismo craniano, traumatismo não especificado.

e. Má formação congênita: Síndrome de DiGeorge, esclerose tuberosa, Síndrome de Rett, displasia ectodérmica, Síndrome de Moebius, Síndrome de Down.

Os procedimentos realizados foram agrupados em invasivos e não invasivos. Sendo os considerados invasivos: exodontias, endodontias, restaurações, raspagem e alisamento radicular. Já os não invasivos incluíram: anamnese, orientação, profilaxia, escovação e aplicação tópica de flúor.

Após a obtenção dos dados, estes foram tabulados e, após análise descritiva, apresentados em forma de tabela com valores absolutos e relativos e figura.

\section{RESULTADOS}

Foram analisados 504 prontuários de pacientes especiais que iniciaram seu tratamento no CEOPE nos anos de 2016, 2017 e 2018. Desses, 46 pacientes se enquadraram no critério de inclusão por terem recebido atendimento em âmbito hospitalar nos anos referidos. Os pacientes atendidos em ambiente hospitalar eram em sua maioria do sexo masculino 28 (60,86\%). A faixa etária mais prevalente foi adulta (entre 19 e 59 anos) com 31 pacientes (67,39\%). A condição clínica prévia mais frequente entre os pacientes foi Doenças crônicas e síndromes metabólicas com 14 pacientes (30,43\%). A maior parte dos pacientes não dispunha de cuidados em regime de Home Care 43 (93,47\%). O tipo da anestesia mais utilizado foi a anestesia geral, em 29 pacientes $(63,04 \%)$. Dos procedimentos realizados em ambiente hospitalar, houve prevalência de procedimentos invasivos, em 30 casos $(65,21 \%)$. Os dados estão apresentados na Tabela 1. 
Tabela 1 - Descrição do perfil dos pacientes atendidos em ambiente hospitalar nos anos de 2016 a 2018

\begin{tabular}{|c|c|c|c|c|c|c|c|}
\hline \multirow{2}{*}{ Características } & \multicolumn{2}{|c|}{2016} & \multicolumn{2}{|c|}{2017} & \multicolumn{2}{|c|}{2018} & \multirow{2}{*}{$p$} \\
\hline & $\mathrm{N}$ & $\%$ & $\mathrm{~N}$ & $\%$ & $\mathrm{~N}$ & $\%$ & \\
\hline \multicolumn{8}{|l|}{ Sexo } \\
\hline Masculino & 10 & 55,5 & 5 & 50 & 13 & 72,2 & \multirow{2}{*}{$-0,431$} \\
\hline Feminino & 8 & 44,5 & 5 & 50 & 5 & 27,8 & \\
\hline \multicolumn{8}{|l|}{ Faixa Etária } \\
\hline 0 a 12 & 0 & 0 & 0 & 0 & 1 & 5,5 & \multirow{4}{*}{0,386} \\
\hline 13 a 18 & 0 & 0 & 1 & 10 & 1 & 5,5 & \\
\hline 19 a 59 & 11 & 61,1 & 6 & 60 & 14 & 77,8 & \\
\hline 60 ou mais & 7 & 38,9 & 3 & 30 & 2 & 11,2 & \\
\hline \multicolumn{8}{|l|}{ Condição Clínica } \\
\hline Doenças Crônicas e Síndromes Metabólicas & 8 & 44,4 & 2 & 20 & 4 & 22,2 & \multirow{5}{*}{0,235} \\
\hline Condição de déficit cognitivo & 4 & 22,2 & 2 & 20 & 5 & 27,8 & \\
\hline Doenças Psíquicas & 1 & 5,6 & 5 & 50 & 4 & 22,2 & \\
\hline $\begin{array}{l}\text { Sequelas físicas permanentes e alterações } \\
\text { sensoriais }\end{array}$ & 5 & 27,8 & 1 & 10 & 4 & 22,2 & \\
\hline Má formação congênita & 0 & 0 & 0 & 0 & 1 & 5,6 & \\
\hline \multicolumn{8}{|l|}{ Home Care } \\
\hline $\operatorname{Sim}$ & 2 & 11,1 & 1 & 10 & 0 & 0 & \multirow{2}{*}{0,354} \\
\hline Não & 16 & 88,9 & 9 & 90 & 18 & 100 & \\
\hline \multicolumn{8}{|l|}{ Tipo de Anestesia } \\
\hline Não Informado & 1 & 5,6 & 0 & 0 & 1 & 5,6 & \multirow{4}{*}{$-0,003$} \\
\hline Não Realizado & 11 & 61,1 & 0 & 0 & 2 & 11,1 & \\
\hline Geral & 6 & 33,3 & 10 & 100 & 13 & 72,2 & \\
\hline Local & 0 & 0 & 0 & 0 & 2 & 11,1 & \\
\hline \multicolumn{8}{|l|}{ Procedimentos Realizados } \\
\hline Invasivos & 7 & 38,9 & 8 & 80 & 15 & 83,3 & \multirow{2}{*}{$-0,011$} \\
\hline Não Invasivos & 11 & 61,1 & 2 & 20 & 3 & 16,7 & \\
\hline
\end{tabular}

Teste $X^{2}$ com nível de significância de $5 \%$.

Destaca-se que, além dos 46 pacientes citados, outros 25 pacientes foram encaminhados para outras unidades hospitalares, sendo 22 para o Hospital Geral para tratamento odontológico sob anestesia geral e 3 para o Hospital do Câncer, para tratamento de lesão de boca. Esses pacientes foram excluídos do trabalho uma vez que em seus prontuários inexistem informações acerca dos tratamentos realizados no ambiente hospitalar, diferentemente do que ocorre com os pacientes encaminhados ao Hospital Metropolitano.

\section{DISCUSSÃO}

Foi observado neste estudo que os pacientes mais frequentemente atendidos em ambiente hospitalar foram aqueles do sexo masculino, com doenças crônicas e síndromes metabólicas, na faixa etária adulta (entre 19 e 59 anos). Também se notou que esses pacientes, na maioria das vezes são atendidos sob anestesia geral para a realização de procedimentos invasivos. Em levantamento semelhante conduzido pelo Departamento de Odontologia Social e Preventiva da Universidade Federal de Minas Gerais $^{6}$, o perfil do paciente com necessidade especial atendido sob sedação e/ou anestesia geral foi parecido com o encontrado neste estudo. Em ambos achados a assistência foi realizada majoritariamente em pacientes adultos, do sexo masculino, oriundos de todas as regiões do estado em que o levantamento foi realizado ${ }^{6}$.
Acredita-se que o número mais elevado de tratamentos odontológicos realizados em pacientes especiais do sexo masculino, na fase adulta, esteja relacionado ao fato de que nesta fase o paciente aumenta em tamanho e força física a um ponto que a execução das técnicas de manejo comportamental não promove o controle adequado que o cirurgião-dentista necessita para realizar seu trabalho, ou mesmo pela maior interferência de movimentos involuntários ${ }^{7}$. Essa resistência do paciente por vezes se estende inclusive para família/cuidadores realizarem os cuidados contínuos de manutenção de saúde bucal, o que eleva a necessidade de tratamentos reparadores serem realizados ${ }^{2}$. Essas hipóteses, no entanto, precisam ser confirmadas em estudos futuros.

Neste estudo os pacientes com doenças crônicas e síndromes metabólicas foram os mais frequentes entre os atendidos no ambiente hospitalar, divergindo de outros estudos como o feito por Santos et al. ${ }^{6}$ e Castro et al. ${ }^{7}$. Talvez essa diferença tenha ocorrido devido ao fato de que neste estudo pacientes portadores de Síndrome de Down não tenham entrado no grupo de pacientes com déficit cognitivo o que pode ter acontecido nos demais trabalhos.

A maioria dos pacientes atendidos em ambiente hospitalar foram submetidos a procedimentos invasivos. Isso pode ter ocorrido em função da dificuldade de seu atendimento em ambiente ambulatorial, as necessidades de tratamento podem ter se agravado e acumulado ${ }^{4}$. Outra possibilidade é o fato de o CEOPE ser um centro de referência para atendimentos de pacientes especiais do estado de Mato Grosso, Acre e Rondônia ${ }^{8}$. Assim, para melhorar a acessibilidade, pode-se ter optado pela realização de tratamento em uma única sessão hospitalar ao invés de várias sessões ambulatoriais, considerando a grande distância a ser percorrida por pacientes procedentes de outros estados ou de municípios do interior de Mato Grosso.

Dado o baixo volume de atendimentos nos três anos estudados, e a complexidade envolvida na realização de procedimentos invasivos no perfil de paciente encontrado pelo estudo, percebe-se a necessidade de mais estudos com essa clientela para saber se ela está sendo efetivamente contemplada pelo Sistema Único de Saúde.

\section{CONCLUSÃO}

O estudo revelou que o perfil do paciente com deficiência ou outra necessidade especial, atendido em ambiente hospitalar é adulto, do sexo masculino, com doenças crônicas e síndromes metabólicas para a realização de procedimentos invasivos sob anestesia geral.

\section{REFERÊNCIAS}

01. Glassman P. A review of guidelines for sedation, anesthesia, and alternative interventions for people with special needs. Spec Care Dentist. 2009; 29(1): 9-16.

02. Chen CY, Chen YW, Tsai TP, Shih WY. Oral health status of children with special health care needs receiving dental treatment under general anesthesia at the dental clinic of Taipei Veterans General Hospital in Taiwan. J Chin Med Assoc. 2014; 77(4): 198-202.

03. Alsaleh I, Cousson PY, Nicolas E, Hennequin M. Is endodontic treatment performed under general anaesthesia technically acceptable? Clin Oral Investig. 2012; 16(6): 1599-606.

04. Sara, NM. Program of dental assistance to special patients: a 13-year experience. RGO. 2011; 59(3): 379-385. 
05. Secretaria de Estado de Saúde. Mato Grosso. [acesso 07 de outubro de 2017]. Disponível em: http://www.saude.mt.gov.br/ceope/ pagina/305/quem-somos\&gt.

06. Santos JS, Valle DA, Palmier AC, Amaral JHL, Abreu MHNG. Availability of hospital dental care services under sedation or general anesthesia for individuals with special needs in the Unified Health System for the State of Minas Gerais (SUS-MG), Brazil. Ciência \&
Saúde Coletiva. 2015; 20(2): 515-524.

07. Castro AM, Marchesoti MGN, Oliveira FS, Novaes MSP. Analysis of dental treatment provided under general anesthesia in patients with special needs. Rev Odontol UNESP. 2010; 39(3): 137-142.

08. Assembleia Legislativa. Mato Grosso. [acesso 09 de junho de 2019]. Disponível em: https://www.al.mt.gov.br/midia/texto/ daltinho-quer-ceope-em-barra-do-garcas/visualizar.

\section{ABSTRACT}

Objective: This study aimed to analyze the profile of the special patients of a dental center treated in a hospital environment. Material and method: The research was conducted through the analysis of medical records of special patients attended in 2016, 2017 and 2018 at the State Center of Dentistry for Special Patients (CEOPE), Cuiabá, MT, Brazil. The parameters analyzed were: gender (male and female), age, previous clinical condition (metabolic diseases; cognitive defect condition; psychic disorders; permanent physical sequelae and congenital malformation), type of anesthesia (general, local, not performed), and procedures performed (invasive; noninvasive; both), and whether or not care was received at home. Results: The patients treated in the hospital environment were mostly male 28 (60.86\%). The most prevalent age group was adult (between 19 and 59 years old) with 31 patients $(67.39 \%)$. The most common prior clinical condition among patients was chronic diseases and metabolic syndromes with $14(30.43 \%)$. Most patients did not have home care assistance 43 (93.47\%). The most used type of anesthesia was general anesthesia in 29 patients $(63.04 \%)$. Of the procedures performed, there was a prevalence of invasive procedures in 30 cases $(65.21 \%)$. Conclusions: The study revealed that the profile of patients with special needs treated in a hospital environment is an adult male with chronic diseases and metabolic syndromes for invasive procedures under general anesthesia.

Keywords: Dental Staff, Hospital; Community Dentistry; Disabled Persons; Dental Care; Dental Service, Hospital.

\author{
AUTOR PARA CORRESPONDÊNCIA \\ Luiz Evaristo Ricci Volpato \\ Rua Estevão de Mendonça, 317, apto 501, Goiabeiras \\ Cuiabá, MT, Brasil. CEP: 78032-085 \\ Telefone: +55 65 98114-5244 \\ E-mail: odontologiavolpato@uol.com.br
}

